\title{
Urologische Langzeitbetreuung von Patienten mit Meningomyelozele II. Therapie
}

\author{
W. F. Thon, J. Denil, C. G. Stief, U. Jonas
}

Urologische Klinik der Medizinischen Hochschule Hannover

\section{Zusammenfassung}

Verschiedene urologische therapeutische Möglichkeiten stehen für Patienten mit Meningomyelozele zur Verfügung. Die jeweilige Therapie muß sich nach der gegenwärtigen Situation der Blasenfunktionsstörung, der Nierenfunktion und dem Kontinenzstatus richten. Eine Nierenfunktionsverschlechterung kann durch einen intermittierenden Einmalkatheterismus oder eine temporäre Vesikostomie bei Neugeborenen verhindert werden. Kontinenzerhaltende Maßnahmen beinhalten die medikamentöse Behandlung mit Anticholinergika, die Blasenerweiterungsplastik und einen gleichzeitig durchzuführenden intermittierenden Einmalkatherismus. Bei Patienten mit Streß-Inkontinenz kann ein artifizieller Schließmuskel implantiert werden. Auch die Sexualfunktion und Fertilität der Patienten mit Meningomyelozele müssen bei den therapeutischen Überlegungen mitberücksichtigt werden.
\end{abstract}

Die soziale Integration von Patienten mit Meningomyelozele ist abhängig von der erfolgreichen Behandlung der Harn- und Stuhlinkontinenz und der orthopädischen Rehabilitation des Gehvermögens bzw. der selbständigen Fortbewegung. Die Lebenserwartung wird heute im wesentlichen durch die frühzeitige urologische Behandlung der neurogenen Blasenfunktionsstörung und der damit zusammenhängenden Nierenfunktion bestimmt. Aus zahlreichen retrospektiven Untersuchungen wissen wir, daß langfristig die Nierenfunktion von der „Blasencompliance“, dem intravesikalen Speicherdruck und dem Detrusor-Sphinkter-Verhalten abhängt $(8,45,49,80,93)$. Das urologische Behandlungskonzept sollte in ständigem Dialog mit den Kollegen der anderen Fachdisziplinen (Pädiater, Orthopädie, Neurochirurg), die den Patienten mitbetreuen, abgestimmt werden.

In der Therapie der neurogenen Blasenfunktionsstörung bei Patienten mit MMC hat sich im Laufe

\section{Urologic Longterm Surveillance of Patients with Meningomyelocele. II. Treatment}

Various urologic treatment options exist and have to be adapted to the current situation of bladder dysfunction, kidney function and continence status in patients with myelomeningocele. Upper urinary tract deterioration can be avoided by intermittent catheterization or temporary vesicostomy in newborns. Procedures to achieve continence include anticholinergics, bladder augmentation and intermittent catheterization or placement of an artificial sphincter in cases of stress incontinence. In addition, sexual function and fertility are nowadays issues which have to be taken into consideration when planning the therapy of patients with meningomyelocele.

\section{Key words}

Meningomyelocele - Genitourinary tract dysfunction - Treatment options der Jahre ein Wandel vollzogen. In den 50er Jahren wurde auch bei nicht dilatiertem oberen Harntrakt bei harninkontinenten Patienten primär eine supravesikale Harnableitung in Form eines Ileum-Conduits durchgeführt. Der dilatierte obere Harntrakt wurde temporär durch eine Ureterostomie oder die von Blocksum beschriebene Vesikokutaneostomie entlastet (37). Die Anlage eines IleumConduits sollte heute nicht mehr als primäre Therapie durchgeführt werden, da wir aus Langzeituntersuchungen wissen, daß bei einem Beobachtungszeitraum von mehr als 10 Jahren in bis zu $60 \%$ der Fälle mit einer Nierenfunktionsverschlechterung zu rechnen ist (29).

Im Vordergrund der urologischen Therapie steht heute die anticholinerge medikamentöse Behandlung einer Detrusorhyperreflexie in Verbindung mit einem intermittierenden Einmalkatheterismus, der als sauberer intermittierender Einmalkatheterismus 1972 von Lapides $\mathrm{u}$. Diokno (72) beschrieben wurde.

Die Blasenentleerung mittels Crédé-Handgriff sollte heute nur noch empfohlen werden, wenn keine funktionelle infravesikale Obstruktion vorliegt, und der 
"Leak-Point-Pressure" höchstens $10-15 \mathrm{cmH}_{2} \mathrm{O}$ beträgt (81).

\section{Pharmakotherapie}

Eine rationale Pharmakotherapie einer Blasenfunktionsstörung sollte nur unter Kenntnis der zugrunde liegenden Pathophysiologie und der pharmakologischen Eigenschaften der verordneten Substanzen erfolgen.

\section{Detrusorhyperreflexie}

Da die bei MMC-Patienten bekannte Gefahr der Nierenfunktionsverschlechterung hauptsächlich auf hohe intravesikale Drucke und eine verminderte "Compliance" zurückzuführen ist, besteht das Ziel der Therapie in der Dämpfung der Detrusorhyperreflexie und Verbesserung der "Compliance". Die Detrusorkontraktilität wird in vitro vermindert durch Anticholinergika, muskulotrope Relaxantien, Kalziumantagonisten und trizyklische Antidepressiva. Wegen fehlender In-vivo-Wirkung oder ausgeprägter Nebenwirkungen einzelner Substanzen stehen für die klinische Anwendung hauptsächlich Anticholinergika (Tab. 1) zur Verfügung $(3,10)$. Die Wirksamkeit der Anticholinergika ist sehr unterschiedlich. Dies beruht im wesentlichen auf der im allgemeinen schlechten Resorption im Gastrointestinaltrakt. In vielen Fällen zwingen dabei die anticholinergen Nebenwirkungen dazu, die Therapie abzusetzen. Oxybutyninchlorid (Dridase ${ }^{(\mathbb{B})}$ führt im Vergleich mit Propanthelin und Plazebo zu einer signifikanten Reduzierung der Detrusorhyperreflexie (112). Allerdings geht die bessere Bioverfügbarkeit und Wirkung von Oxybutyninchlorid mit einer höheren Rate anticholinerger Nebenwirkungen einher. Diese Nebenwirkungen sind durch die topische Applikation von Oxybutyninchlorid in Verbindung mit einem intermittierenden Einmalkatheterismus zu vermeiden $(24,54)$. 5-mg-Tabletten werden in einem Mörser zerkleinert und mit $10 \mathrm{ml}$ steriler Kochsalzlösung zweimal täglich transurethral in die Blase instilliert. Intravesikal verabreichtes Oxybutyninchlorid wird schnell resorbiert und führt zu höheren Plasmakonzentrationen als bei oraler Applikation. Trotzdem treten bei intravesikaler Gabe weniger Nebenwirkungen auf als bei oraler Einnahme, es sei denn, daß es sich um einen Zustand nach Blasenaugmentation handelt und die Substanz über die Darmschleimhaut resorbiert wird (78). Obwohl vom Hersteller darauf hingewiesen wird, Dridase ${ }^{(\mathbb{B})}$ erst ab dem 5. Lebensjahr einzusetzen, wurde Oxybutyninchlorid erfolgreich und ohne Nebenwirkungen bereits bei Neugeborenen angewendet $(7,61,116)$. Während Baskin u. Mitarb. (7) eine Dosierung von $0,1 \mathrm{mg} / \mathrm{kg} \mathrm{KG}$ zweimal täglich als Dauermedikation empfehlen, geben Wang u. Mitarb. (116) sogar eine Dosierung von 0,36 bis $0,5 \mathrm{mg} / \mathrm{kg} \mathrm{KG} / \mathrm{die}$ an.

\section{Tab. 1}

\begin{tabular}{lll}
\hline Freiname & Handelsname & Dosierung/die \\
\hline Oxybutyninchlorid* & Dridase $^{\circledR}$ & $2 \times 0,1-0,25 \mathrm{mg} / \mathrm{kg} \mathrm{KG}$ \\
Propanthelinbromid & Corrigast $^{\circledR}$ & $4 \times 15-30 \mathrm{mg}$ \\
Metanthelinbromid & Vagantin $^{\circledR}$ & $3 \times 25-50 \mathrm{mg}$ \\
Trospiumchlorid & Spasmex $^{\circledR}$ & $3 \times 2,5-5 \mathrm{mg}$ \\
\hline
\end{tabular}

* topische Applikation. $2 \times 5 \mathrm{mg}$ in $10 \mathrm{ml} \mathrm{NaCl}$-Lösung

\section{Urethraverschlußinsuffizienz}

Eine medikamentöse Stimulation der alpha-Rezeptoren des Blasenhalses und der proximalen Harnröhre mit Sympathomimetika führt zwar bei der Urethraverschlußinsuffizienz zu einer urethralen Widerstandserhöhung, doch sind die Nebenwirkungen durch Stimulation des sympathischen Nervensystems häufig sehr ausgeprägt und zwingen zum Absetzen des Medikamentes.

\section{Funktionelle infravesikale Obstruktion}

Die funktionelle infravesikale Obstruktion am Blasenhals und in der proximalen Harnröhre ist effektiv durch Gabe von alpha-Rezeptorenblockern zu therapieren. Phenoxybenzamin (Dibenzyran ${ }^{\circledR}$ ) und Prazosin (Minipress $^{(\circledR)}$ sind die bekanntesten Medikamente. Die klassischen Nebenwirkungen wie orthostatische Hypotension, Tachykardie und Nasenschleimhautschwellung können durch Verordnung eines selektiven $\alpha 1$-Rezeptorenblockers wie Prazosin (Minipress ${ }^{\circledR}$ ) oder Doxazosinmesilat (Diblo$\operatorname{cin}^{(\mathbb{B})}$ vermieden werden. Nach Amark u. Nergardh (2) haben alpha-Rezeptorenblocker zusätzlich eine dämpfende Wirkung auf die Detrusorhyperreflexie.

Eine Überaktivität im Sphinkter externusBereich kann durch zentral muskelrelaxierende Substanzen wie Baclophen (Lioresal $\left.{ }^{\circledR}\right)$ oder dem direkt peripher an der quergestreiften Muskulatur angreifenden Dantrolen $\left(\right.$ Dantamacrin $\left.{ }^{\circledR}\right)$ therapiert werden. Der klinische Einsatz dieser Substanzen ist dadurch limitiert, daß sie nicht spezifisch an Blase, Harnröhre und SchlieBmuskel wirken und ihre Nebenwirkungen häufig zum Absetzen der Medikation zwingen.

\section{Sauberer intermittierender Einmal- katheterismus}

In der Therapie der funktionellen infravesikalen Obstruktion bei MMC-Patienten steht der saubere intermittierende Einmalkatheterismus an erster Stelle. Nach der Einführung des intermittierenden Einmalkatheterismus ("Clean Intermittent Catheterization" = CIC) durch $L a$ pides u. Diokno (72), wurde diese Behandlungsmethode bei bestimmten Formen der neurogenen Blase bei Kindern und Erwachsenen schon bald die Therapie der Wahl (61). Für den sauberen intermittierenden Einmalkatheterismus bei Neugeborenen und Kindern stehen pädiatrische Ernährungssonden der Größen Ch. 8-12 mit einem Gleitmittel oder besonders gleitende Katheter, die aus Polyvinylpyrrolidon mit speziell wasserbindenden Eigenschaften gefertigt sind (Lofric ${ }^{\circledR}$ Katheter), der Größen Ch. 8-14 mit gerader oder Tiemannspitze, zur Verfügung. Um die Compliance der Eltern und der Familie für diese Therapiemaßnahme zu verbessern, ist es erforderlich, die Durchführung und die Notwendigkeit des Katheterismus genauestens zu erklären und sie in der Technik bestens zu schulen (55). Erfahrungsgemäß sind Kinder etwa ab dem 4. -6 . Lebensjahr bei entsprechender Schulung in der Lage, den Katheterismus selbständig durchzuführen (60).

Van Gool u. Mitarb. (113) konnten bei einem Follow-up von 45 Monaten zeigen, daß bei 61 mit dem Einmalkatheterismus behandelten Kindern die Inzidenz von 
Tab. 2 Nierenfunktionsverschlechterung (NFV) bei Detrusor-SphinkterDyssynergie: Exspektatives Verhalten vs. intermittierender Einmalkatheterismus und Anticholinergika (63).

\begin{tabular}{lcc}
\hline & \multicolumn{2}{c}{ Nierenfunktionsverschlechterung } \\
$\%$ & $n$ & 8 \\
\hline $\begin{array}{l}\text { Intermitt. EK, Anticholinergika } \\
\text { exspektativ }\end{array}$ & $2 / 26$ & 8 \\
\hline
\end{tabular}

Harnwegsinfekten signifikant abnahm und die Kontinenzrate signifikant zunahm. Adjuvante Therapiemaßnahmen bestanden in einer anticholinergen Therapie bei Detrusorhyperaktivität und einer Blasenhalssuspension (Kolposuspension) bei Sphinkterhypoaktivität und Urethraverschlußinsuffizienz. Geraniotis u. Mitarb. (47) konnten nachweisen, daß sich bei MMC-Patienten mit DetrusorSphinkter-Dyssynergie, bei denen bei Spontanmiktion im Laufe der Zeit in $50 \%$ der Fälle mit einer Verschlechterung der Nierenfunktion zu rechnen ist, bei Durchführung des intermittierenden Einmalkatheterismus eine Nierenfunktionseinschränkung nur bei $10 \%$ der Patienten auftritt. Diese Ergebnisse wurden durch Kasabian et al. (63) bestätigt, die bei Detrusor-Sphinkter-Dyssynergie oder spastischem Beckenboden und bereits im Neugeborenenalter begonnenem intermittierenden Einmalkatheterismus und anticholinerger Therapie eine Nierenfunktionsverschlechterung nur bei $8 \%$ der Kinder feststellten. Im Gegensatz dazu war in einem exspektativ behandelten Patientengut eine Nierenfunktionsverschlechterung in $48 \%$ der Fälle nachzuweisen (Tab. 2). Lindehall et al. (73) sahen bei 17 von 27 Kindern mit bereits bestehenden sekundären Harnstauungsnieren oder einem sekundären Reflux bei Durchführung des intermittierenden Einmalkatheterismus eine Normalisierung des oberen Harntrakts. Allerdings zeigte sich keine Befundverbesserung oder sogar eine Nierenfunktionsverschlechterung bei $50 \%$ der Kinder mit bereits bestehender Dilatation und höhergradigem Reflux zu Beginn des intermittierenden Einmalkatheterismus.

Die Dilatation des oberen Harntraktes kann sich nach Aufnahme eines intermittierenden Einmalkatheterismus innerhalb weniger Monate vollständig zurückbilden (Abb. 1 a u. b). Koch et al. (69) verglichen 63 MMC-Patienten, die über 12-22 Jahre einen intermittierenden Einmalkatheterismus durchführten mit 30 MMC-Patienten, bei denen vor 17-23 Jahren eine supravesikale Harnableitung in Form eines Ileum-Conduits angelegt wurde: In der Conduit-Gruppe traten signifikant häufiger Pyelonephritiden ( $21 \%$ vs. $60 \%$ ), Nierenfunktionsverschlechterung ( $8 \%$ vs. $57 \%$ ) und eine Nephrolithiasis ( $2 \%$ vs. $43 \%$ ) auf (Tab. 3).

Tab. 3 Nierenfunktionsverschlechterung (NFV), Pyelonephritiden (PN) und Nephrolithiasis (NL) bei intermittierendem Einmalkatheterismus oder nach lleum-Conduit-Anlage (69).

\begin{tabular}{lllllr}
\hline & $\begin{array}{l}\text { Follow-up } \\
\text { Jahre }\end{array}$ & $\mathrm{n}$ & $\begin{array}{l}\text { NFV } \\
\%\end{array}$ & $\begin{array}{c}\text { PN } \\
\%\end{array}$ & $\begin{array}{c}\text { NL } \\
\%\end{array}$ \\
\hline intermitt. EK & $12-22$ & 63 & 8 & 21 & 2 \\
Ileum-Conduit & $17-23$ & 30 & 57 & 60 & 43 \\
\hline
\end{tabular}
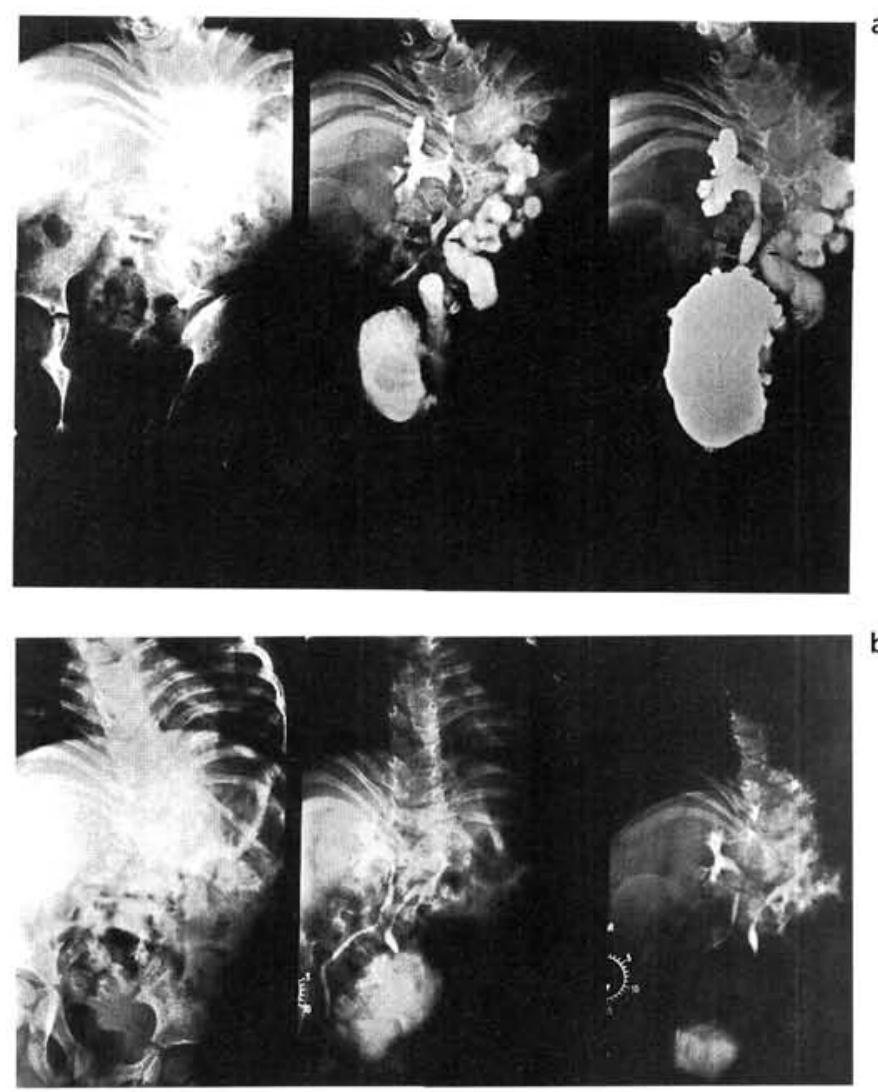

Abb. 1 a IVP vor Beginn eines intermittierenden Einmalkatheterismus: bIVP 3 Monate nach Beginn des intermittierenden Einmalkatheterismus.

Daß der intermittierende Einmalkatheterismus auch bei Neugeborenen in der überwiegenden Zahl der Fälle ohne wesentliche Nebenwirkungen durch die Eltern durchgeführt werden kann, wurde in mehreren Studien bestätigt $(7,47,59)$.

\section{Komplikationen des intermittierenden Einmalkatheterismus}

Über Komplikationen des sauberen intermittierenden Einmalkatheterismus wird in der Literatur selten berichtet (Tab. 4). Thirumavalavan u. Ransley (110) dokumentierten in ihrem Krankengut eine Epididymitis bei $14 \%$ der Kinder und jungen Erwachsenen, die wegen einer neurogenen Blase einen intermittierenden Einmalkatheterismus durchführten.

Tab. 4

\begin{tabular}{|c|c|c|c|}
\hline Komplikationen & $n$ & $\%$ & Autoren \\
\hline $\begin{array}{l}\text { signif. Bakteriurie } \\
\text { symptomatisch }\end{array}$ & $\begin{array}{l}43 / 50 \\
16 / 43\end{array}$ & $\begin{array}{l}86 \\
37\end{array}$ & Perkash, Giroux, 1993* \\
\hline Epididymitis & $10 / 99$ & 14 & Thirumavalan, Ransley, 1992 \\
\hline $\begin{array}{l}\text { Blasenperforation } \\
\text { nach Augmentation, } \\
\text { bei EK }\end{array}$ & $\begin{array}{l}12 / 264 \\
11 / 12\end{array}$ & $\begin{array}{l}4,5 \\
90\end{array}$ & Bauer et al., 1992 \\
\hline
\end{tabular}

* 13,63 Infektionen/1 000 Patiententage 
Eine signifikante Bakteriurie tritt bei Durchführung des sauberen intermittierenden Einmalkatheterismus bei etwa $90 \%$ der Patienten auf, in einem Drittel der Fälle mit klinischer Symptomatik. Mit 14 Infektionen pro 1000 Patientage ist die Inzidenz einer Bakteriurie aber insgesamt gering (94). Bei rezidivierenden Harnwegsinfekten oder bekanntem Reflux bei Aufnahme des Katheterismus sollte eine niedrig dosierte antibiotische Dauerprophylaxe mit Nitrofurantoin (Furadantin ${ }^{\circledR}$ ) oder Cotrimoxazol (Cotrim ${ }^{\circledR}$, Bactrim ${ }^{\circledR}$, Eusaprim $\left.{ }^{\circledR}\right) 1-2 \mathrm{mg} / \mathrm{kg}$ KG/Tag durchgeführt werden. Symptomatische Harnwegsinfekte mit Fieber müssen durch eine adäquate resistenzgerechte Antibiotikatherapie behandelt werden. Um weitere strukturelle Schädigungen der Blasenwand zu verhindern, sollten auch asymptomatische Bakteriurien testentsprechend behandelt werden.

Eine besonders sorgfältige Schulung der Eltern und der Kinder in der Durchführung des intermittierenden Einmalkatheterismus ist wegen der Gefahr einer Blasenperforation erforderlich, wenn eine Blasenaugmentation durchgeführt wurde. Bauer u. Mitarb. (11) sahen in ihrem Gesamtkrankengut blasenaugmentierter Kinder eine Perforation in 12 Fällen (4,5\%). 11 dieser 12 Kinder führten einen sauberen intermittierenden Einmalkatheterismus durch.

\section{Vesikokutaneostomie}

Ist insbesondere bei kleinen Jungen die Durchführung eines Einmalkatheterismus durch die Eltern nicht möglich, kann zur Überbrückung der Zeit, bis der Junge selbst dazu in der Lage ist, eine Vesikokutaneostomie angelegt werden, die einfach mit einer Windel versorgt wird $(37,77)$. Insbesondere bei dilatiertem oberen Harntrakt und Problemen bei der Durchführung des intermittierenden Einmalkatheterismus (Compliance, rez. fieberhafte Harnwegsinfekte trotz Langzeitantibiose) sollte man sich frühzeitig für die Anlage einer Vesikokutaneostomie entscheiden. Der spätere Verschluß der Vesikostomie ist unkompliziert und sollte dann durchgeführt werden, wenn das Kind mental in der Lage ist, sich selbst zu katheterisieren und den Wunsch hat, kontinent zu sein. Die funktionelle Blasenkapazität erholt sich in Abhängigkeit von der Detrusorhyperreflexie bis zu altersentsprechenden Werten.

\section{Appendikovesikostomie}

Ist auch in späterem Lebensalter ein intermittierender Einmalkatheterismus wegen Schmerzen oder Komplikationen in Form einer Via falsa oder rezidivierender Epididymitiden nicht möglich oder die mehrmals tägliche Durchführung des Katheterisierens bei auf den Rollstuhl angewiesenen Patienten zu beschwerlich, kann eine Appendikovesikostomie im Unterbauchbereich nach $\mathrm{Mi}$ trofanoff angelegt werden, um einen Selbstkatheterismus unter Umgehung der Harnröhre zu ermöglichen $(66,87)$. Borzi et al. (22) berichtete über eine $80 \%$ ige Erfolgsrate bei 10 Kindern mit Appendicovesikostomie.

In Abhängigkeit von der urodynamischen Untersuchung und der Ausprägung der Harninkontinenz sollte bei Patienten mit niedriger "Compliance" und hoher intravesikaler Druckamplitude immer ein kompletter Bla- senersatz in Form einer kontinenten Harnableitung (KockPouch oder Mainz-Pouch I mit Nabelstoma) diskutiert werden (s. unten). Bei Anlage eines Mainz-Pouches oder eines anderen ileozökalen Pouches kann die Appendix als katheterisierbares Stoma verwendet werden. Elder (40) berichtete über 13 Patienten mit Blasenexstrophie oder MMC, die nach Anlage eines ileozökalen Reservoirs mit kontinentem Appendixstoma bei einem Follow-up von 2-48 Monate vollständig kontinent waren.

\section{Urethradilatation}

Die urethrale Dilatation (Abb.2) stellt bei Mädchen mit hohen intravesikalen Speicher-Drücken, die durch Anticholinergika und den intermittierenden Einmalkatheterismus nicht effizient behandelt werden können, eine Alternative zu einer temporären Vesikostomie dar $(58,101,116)$. Die Dilatation ist auch bei Neugeborenen ohne Gefahr der SchlieBmuskelverletzung bis $22 \mathrm{Ch}(=\mathrm{He}-$ gar Dilatator Nr.12) durchführbar und kann, falls erforderlich, auch mehrfach wiederholt werden. Bloom et al. (18) berichteten über 18 Kinder, die im Alter von 1 Woche bis zu 9 Jahren bis zu $35 \mathrm{mal}$ dilatiert wurden. Bei einem Follow-up von bis zu 5 Jahren sahen diese Autoren eine Verbesserung der "Compliance" und eine Abnahme des "Leak-Point-Pressure" von $54,67 \mathrm{cmH}_{2} \mathrm{O}$ auf $19 \mathrm{cmH}_{2} \mathrm{O}$ (Tab. 5). Bei Knaben kann die Urethradilatation im Sphinkter externus-Bereich unter fluoroskopischer Kontrolle mit einem Dilatationskatheter bis 24 Ch durchgeführt werden. Die Urethradilatation ist dann als erfolgreich anzusehen, wenn der "Leak-Point Pressure" nach der Dilatation kleiner als $40 \mathrm{cmH}_{2} \mathrm{O}$ ist.

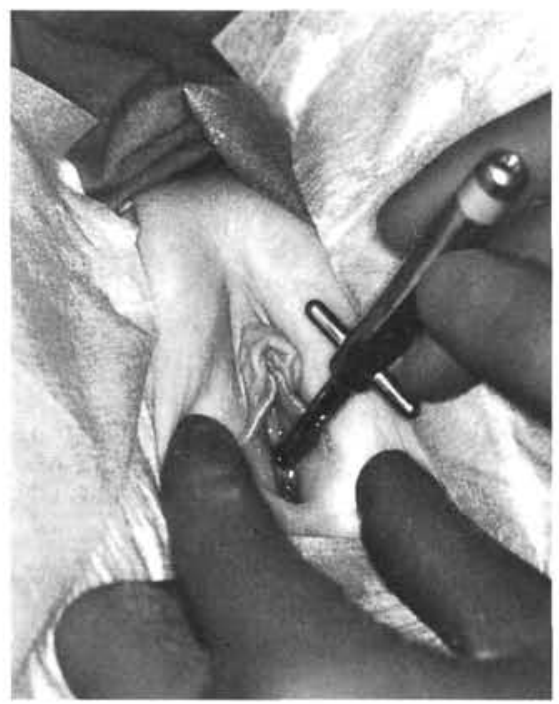

Abb. 2 Urethradilatation mit dem Diddle Bougie.

Tab.5 Einfluß der "Urethradilatation" auf den "Leak-Point Pressure", die Compliance und die maximale Blasenkapazität (18).

\begin{tabular}{lcc}
\hline & Prä-Dilatation & Post-Dilatation \\
\hline "Leak-Point Pressure" $\left(\mathrm{cmH}_{2} \mathrm{O}\right)$ & 54,6 & 19 \\
"Compliance" $\left(\mathrm{ml} / \mathrm{cmH}_{2} \mathrm{O}\right)$ & 11,7 & 27,4 \\
max. Blasenkapazität $(\mathrm{ml})$ & 104,6 & 161,1 \\
\hline
\end{tabular}

Patientengut: 18 Kinder, 35 Dilatationen bis "LPP" $<40 \mathrm{CmH}_{2} \mathrm{O}$

Alter: 1 Woche bis 9 Jahre, Follow-up: bis zu 5 Jahre 
$a, b$

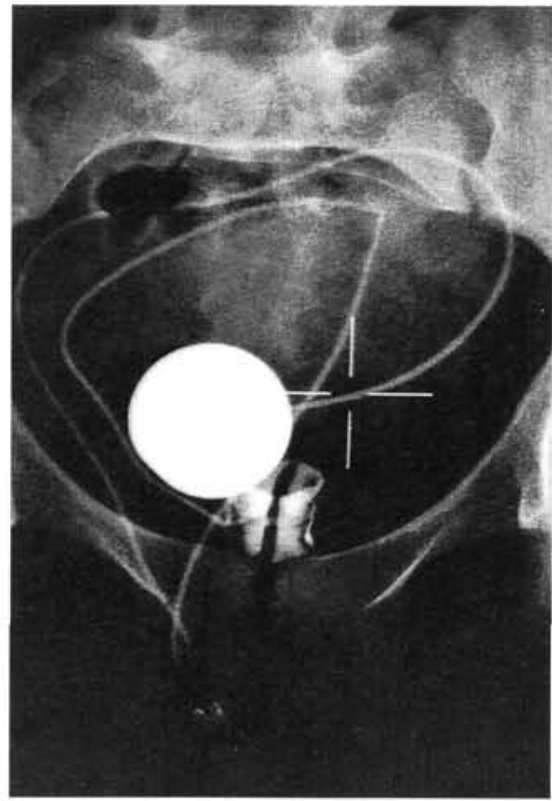

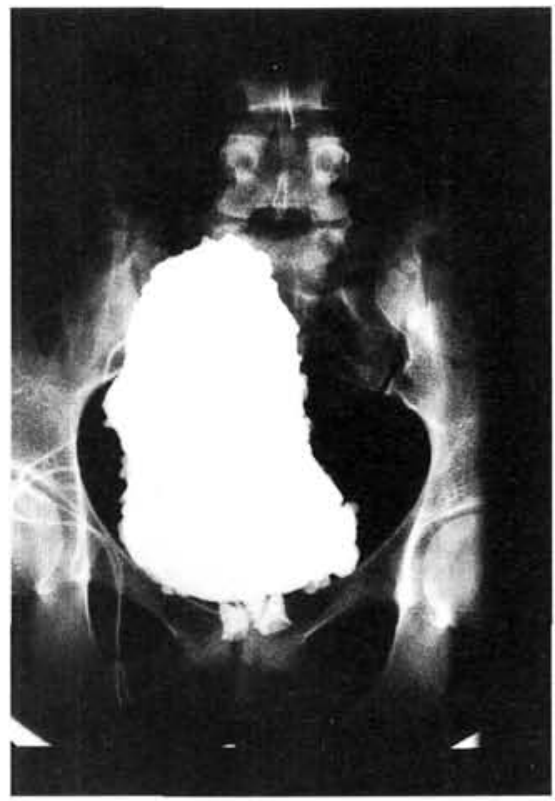

Abb. 3 Artifizieller Sphinkter AMS 800 Manschette implantiert um den Blasenhals, a Leeraufnahme, b Zystogramm.

\section{Artifizieller Schließmuskel}

Bei einer urethralen Verschlußinsuffizienz und einer normalen "Compliance" der Blase besteht nach Ausschluß einer Detrusorhyperreflexie die Möglichkeit der Implantation eines artifiziellen Schließmuskels (AMS $\left.800^{\circledR}\right)$. Hierbei wird die Druckmanschette bei Jungen um die bulbäre Harnröhre oder den Blasenhals, bei Mädchen um den Blasenhals implantiert (Abb. $3 \mathbf{a}$ u. b). Die Kinder sollten mindestens 10 Jahre alt sein, um das System regelrecht bedienen zu können. Nach Zusammendrücken der im Skrotum oder in einer der großen Labien implantierten Pumpe strömt die Flüssigkeit aus der um die Harnröhre gelegten Manschette in das Druckreservoir. Nach 3-4 Minuten fließt die Flüssigkeit automatisch wieder in die Manschette zurück und verschließt die Harnröhre durch Kompression. Revisionseingriffe sind auch bei mit der Methode erfahrenen Operateuren häufig. Barrett u. Parulkar (6) berichten über mechanische und chirurgische Probleme als Revisionsursache in jeweils 18,6\% der Fälle. Eine Arrosion der Manschette und Infektion des implantierten Systems traten in 11,6 \% der Fälle auf. Barrett u. Parulkar (6) führten 24 Reoperationen bei 43 implantierten Kindern durch (0,56/Patient) (Tab. 6). Stephenson (105) berichtete über 23 Harnröhrenarrosionen durch die Manschette in $15 \mathrm{~Pa}$ tienten bei insgesamt 102 implantierten Patienten.

Tab. 6 Revisionsoperationen nach Implantation eines artifiziellen Sphinkters (AMS 800) im Kindesalter (6).

\begin{tabular}{llc}
\hline & \multicolumn{2}{c}{$\begin{array}{c}\text { Revisionsoperationen } \\
\text { n }\end{array}$} \\
\hline mechanische Ursache & 8 & 18,6 \\
chirurgische Gründe & 8 & 18,6 \\
Arrosion u. Infektion & 5 & 11,6 \\
Reimplantationen & 2 & 4,7 \\
\hline Summe & $24 / 43$ & 55,8 \\
\hline
\end{tabular}

Nach der Implantation muß in regelmäßigen Abständen eine sonographische Kontrolle des oberen Harntraktes erfolgen, da bereits mehrfach über eine zunehmende Nierenfunktionseinschränkung infolge neu aufgetretener Harnstauungsnieren oder Refleuxnephropathien berichtet wurde $(9,16)$. Besonders gefährdete Patienten waren Kinder mit bereits präoperativ niedriger "Compliance", kleiner funktioneller Blasenkapazität und einer leichten Detrusorhyperreflexie (Abb.4). Über Jahre kann sich nach Implantation eines artifiziellen Sphinkters eine ausgeprägte Detrusorhyperreflexie mit den sekundären Veränderungen des oberen Harntraktes entwickeln. Therapeutische Maßnahmen beinhalten eine Explantation des Sphinkters, oder eine dekompressive Laminektomie mit Neurolyse bei nachgewiesenem "tethered cord"-Syndrom oder eine Augmentation der Blase.

Trotz der erforderlichen Revisionen ist im Endeffekt mit einer guten Funktion des artifiziellen Sphinkters in $85 \%$ der Fälle zu rechnen (105).

\section{Blasenaugmentation}

Die Indikation für eine Augmentation ist die kleine Blase mit niedriger "Compliance", die trotz anticholinerger Medikation nur eine geringe funktionelle Kapazität bei niedrigen Blasendrucken aufweist, so daß sie nicht als Urinreservoir dienen kann und keine Miktion in sozial akzeptablen Intervallen ermöglicht (46). Voraussetzung für die Augmentation ist, daß der Patient in der Lage ist, die Blase mit einem "Leak-Point Pressure" $<20 \mathrm{cmH}_{2} \mathrm{O}$ zu entleeren oder sich selbst zu katheterisieren. Eine Blasenaugmentation wird mit Hilfe von detubularisierten Dünn- oder Dickdarmanteilen (86), Magen $(1,4)$, oder als sogenannte Detrusormyotomie-Autoaugmentation- (28) bzw. bei bereits einseitig funktionsloser Niere als Ureterozystoplastik $(12,117)$ durchgeführt (Tab. 7). Die von Bramble (23) beschriebene Clam-Zystoplastik stellt eine effektive Behandlungsmethode dar, um die Compliance der Blase und die funktionelle Kapazität zu erhöhen und gleichzeitig den in- 


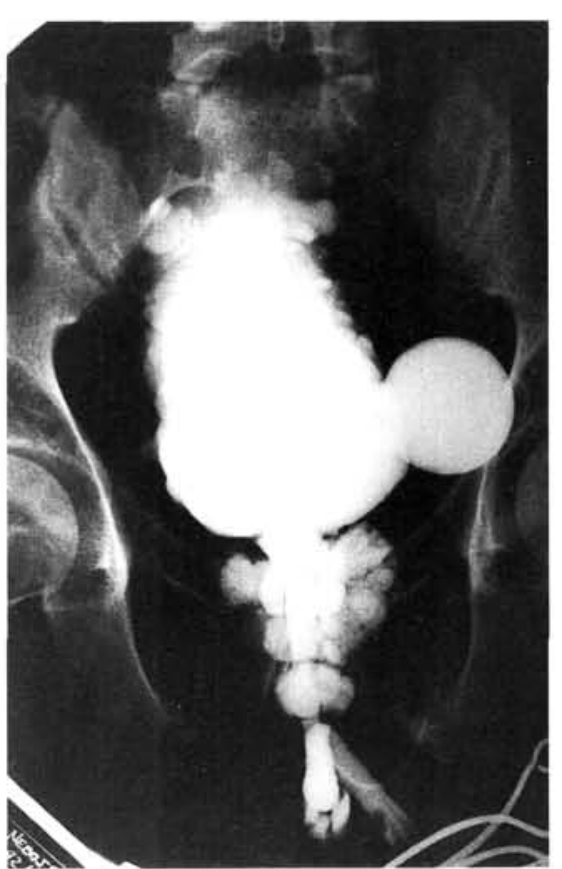

Abb. 4 Artifizieller Sphinkter AMS 800, Manschette implantiert um die bulbäre Harnröhre, Zystogramm bei ausgeprägter Detrusorhyperreflexie mit Pseudodivertikel und Reflux in die Prostatagänge.

travesikalen Druck in tolerable Bereiche zu senken (67). Durch Senkung der intravesikalen Drucke wird einer sekundären Nierenfunktionsverschlechterung präventiv entgegengewirkt (75). Nach den Erfahrungen von Cher u. Allen (31) mit 16 Patienten mit Ileozystoplastik genügt alleine die Augmentation, um eine Kontinenz zu gewährleisten, wenn der urethrale Widerstand mindestens $25 \mathrm{cmH}_{2} \mathrm{O}$ beträgt.

Kontraindikationen für eine intestinale Augmentation stellen entzündliche Darmerkrankungen dar. Mundy u. Nurse (90) und Wagstaff et al. (114) beobachteten bei etwa $20 \%$ ihrer Kolozystoplastiken bzw. ileumaugmentierten Kindern ein verzögertes lineares Wachstum. Als ursächlicher Faktor wurde eine chronische metabolische Azidose mit den entsprechenden Auswirkungen auf den Skelett-Mineralstoffwechsel angesehen, obwohl die Autoren keinen signifikanten Wachstumsunterschied zwischen Kindern mit normalem Säurebasen-Haushalt und Kindern mit metabolischer Azidose feststellen konnten.

Bei bereits bestehender kompensierter Niereninsuffizienz und Azidose sollte keine Dünndarm- oder Dickdarmaugmentation, sondern eine Gastrozystoplastik durchgeführt werden, um eine metabolische Azidose zu vermeiden. Vorteile der Gastrozystoplastik bestehen in niedrigeren Infektionsraten, geringerer Schleimproduktion, leichter Ureterreimplantation und guter Dehnbarkeit

Tab.7 Möglichkeiten der Blasenaugmentation.

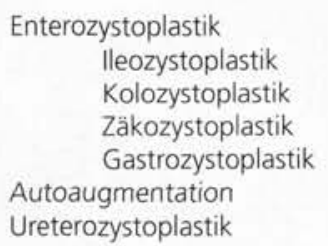

des Segmentes. Gastrointestinale Komplikationen nach Gastrozystoplastik wurden von Gold et al. (52) berichtet, die über postoperative Gedeihstörungen und Gewichtsverlust infolge Nahrungsverweigerung, verzögerter $\mathrm{Ma}$ genentleerung und Ösophagitis berichteten. Aziduriebedingte Probleme der mit Magen augmentierten Blasen wurden von Dykes u. Ransley (38) bei 2 von 8 Kindern gesehen. Kinahan et al. (68) berichteten über ein Kind mit schwerster Zystitis und metabolischer Alkalose verursacht durch die Azidurie. Über ein Kind mit perforiertem peptischen Ulkus in dem magenaugmentierten Teil der Blase wurde von Reinberg et al. (96) berichtet.

Jede Augmentation unter Benutzung intestinaler Segmente geht mit einer mehr oder weniger ausgeprägten Schleimproduktion, einer veränderten Elektrolytabsorption (79) und der Gefahr einer durch einen intermittierenden Einmalkatheterismus verursachten Blasenperforation $(11,39)$ oder selten Spontanperforation (100) einher. Als Ursache einer spontanen Ruptur der augmentierten Blase wird eine Ischämie der Blasenwand in Verbindung mit einer chronischen Überdehnung durch Schleim bedingte Obstruktion angesehen (32). Bei Verdacht auf eine Perforation besteht bei der Durchführung eines Zystogramms mit Kontrastmittel die Gefahr, daß das Kontrastmittel über einen ventrikuloperitonealen Shunt als Reflux in die Ventrikel kommt und $\mathrm{zu}$ einem schweren neurologischen Insult führt (33). Aus diesem Grunde sollte bei entsprechendem Verdacht auf eine Perforation der augmentierten Blase das Zystogramm nur mit nichtionischem Kontrastmittel (z. B. Solutrast ${ }^{\circledR}$, Byk Gulden) durchgeführt werden, um die Neurotoxizität zu vermeiden.

Ileo-, Zöko- und Kolozystoplastik beinhalten das Risiko einer malignen Transformation, in den meisten Fällen im Bereich der enterovesikalen Grenzschicht als Adenokarzinom (42). Nach einer Literaturrecherche von Harzmann (56) wurde weltweit über 32 Harnableitungs-Karzinome berichtet, in 11 Fällen in dünndarm- und in 6 Fällen in dickdarmaugmentierten Blasen.

Auf eine mögliche lithogene Disposition einer Enterozystoplastik weisen Blyth et al. (20) hin. $30 \%$ ihrer darmaugmentierten Patienten hatten Blasensteine, während kein Patient mit Gastrozystoplastik Steine bildete. Bei den Steinen handelte es sich um Infektsteine durch Urease spaltende Bakterien. Dies ist insofern von Bedeutung, da sich nach Untersuchungen von Fenn et al. (41) eine positive Urinkultur nach "Clam"-Zystoplastik bei etwa $80 \%$ der Patienten, die einen intermittierenden Einmalkatheterismus durchführen, und $60 \%$ der Patienten, die spontan miktionieren, nachweisen läßt.

\section{Supravesikale Harnableitung}

In den 60er Jahren wurde bei vielen Patienten mit neurogener Blase auch bei nicht erweitertem oberen Harntrakt ein Ileum- oder Kolon-Conduit angelegt. Seit Einführung des intermittierenden Einmalkatheterismus wird die supravesikale Harnableitung nur noch selten als primäre Maßnahme bei MMC-Kindern durchgeführt. Die in Verbindung mit jeder Form der Harnableitung unter Verwendung von Darmsegmenten auftretende chronische 
hyperchlorämische metabolische Azidose kann zu einer Osteomalazie führen $(69,79)$.

1950 beschrieb Bricker die Methode des Ileum-Conduits. Um rezidivierende Pyelonephritiden nach Ileum-Conduit-Anlage mit Anastomose der Ureteren ohne Antirefluxschutz zu vermeiden, wurde von $M o g g$ (88) als Alternative ein Stück Kolon als Conduit mit antirefluxiver Implantation der Ureteren vorgeschlagen. Koch et al. (69) beobachteten bei 30 Kindern und einem Follow-up von 12-23 Jahren nach Anlage eines Ileum-Conduits rezidivierende Pyelonephritiden bei $60 \%$ und eine Nierenfunktionsverschlechterung bei $57 \%$. Im Vergleich dazu trat eine Nierenfunktionsverschlechterung nur bei $8 \%$ von $63 \mathrm{~Pa}$ tienten auf, die über einen Beobachtungszeitraum von 12 22 Jahren einen sauberen intermittierenden Einmalkatheterismus als Therapie ihrer neurogenen Blasenfunktionsstörung durchführten (Tab. 3). Stomakomplikationen und ureterointestinale Stenosen nach Ileum-Conduit-Anlage, die eine operative Revision erforderlich machten, traten in $37 \%$ bzw. $17 \%$ auf. In einer Literaturübersicht von Cass et al. (29) über Langzeitverläufe von > 10 Jahren nach Conduitanlage im Kindesalter wird eine Nierenfunktionsverschlechterung nach einer Ileum-Conduit-Anlage in $16,5-61 \%$ und nach Anlage eines Kolon-Conduits in $61 \%$ angegeben, obwohl die antirefluxive Implantation der Ureteren in den Dickdarm gerade eine Nierenfunktionsverschlechterung verhindern sollte.

Im gleichen Jahr wie Bricker (25) berichtete Gilchrist (50) über eine ileozökale Pouchtechnik. Da bei seinem Operationsverfahren die Kontinenz allein durch eine kompetente Ileozökalklappe zustande kommen sollte und keine Detubularisierung der Darmabschnitte erfolgte, wurde dieses Verfahren wegen Inkontinenz zum damaligen Zeitpunkt nicht weiter verfolgt.

Heute stellen kontinente Harnableitungen unter Verwendung von Dünn- und/oder Dickdarmabschnitten Standardeingriffe in den meisten urologischen Kliniken dar. Benson et al. (15) verglichen Früh-, Spät- und schwere Komplikationen, Reoperationsraten und operative Mortalität nach Standardharnableitung in Form eines Ileum-Conduit $(\mathrm{n}=51)$ mit Anlage eines Ileozökal-Pouches $(n=22)$. Zwischen beiden Gruppen wurden keine signifikanten Unterschiede festgestellt.

Populäre Methoden der kontinenten Harnableitung sind der Koch-Pouch (70), Mainz-Pouch (111) und der Indiana-Pouch (97).

Bei Verwendung des Ileozäkalsegmentes sollte postoperativ in 6-12 monatigen Abständen der Vitamin $\mathrm{B}_{12}$-Spiegel bestimmt werden. Steiner $\mathrm{u}$. Mitarb. (104) fanden niedrige Vitamin $B_{12}$-Spiegel bei $25 \%$ ihrer Patienten, die einen ileozökalen Blasenersatz bekommen hatten. Ein klinisch evidenter Vitamin $B_{12}$-Mangel ist bei kompletter Malabsorption frühestens nach 3-6 Jahren, bei partieller Malabsorption nach 6-30 Jahren zu erwarten (79).

Bei den kontinenten Harnableitungsformen besteht, ebenso wie bei den darmaugmentierten Blasen, eine Tumorinduktionsgefahr an der ureteroenteralen
Grenzschicht. Die Stase des Urins mit der längeren Einwirkungszeit möglicher kanzerogener Metaboliten könnte zu einer vielfach höheren Tumorinzidenz bei kontinenten als bei inkontinenten Harnableitungen führen. Die übliche Latenzzeit bei allen Formen der Harnableitung über Darmsegmente beträgt etwa 20 Jahre (56). Zytologische und endoskopische Kontrolluntersuchungen nach Anlage einer kontinenten Harnableitung sollten einmal jährlich, beginnend spätestens 5 Jahre nach dem Eingriff, durchgeführt werden.

\section{Neurostimulation}

Die intravesikale transurethrale Elektrostimulation nach Katona (64) hat drei Ziele:

1. das Gefühl für die Blasenfüllung zu erlangen,

2. eine Detrusorkontraktion auszulösen und

3. eine bewußte Kontrolle über die Blasenfunktion zu erreichen (64).

Katona u. Berenyi (64) berichten, daß 71 von 100 mit transurethraler Elektrostimulation behandelte Patienten Tag und Nacht kontinent wurden. Auch $\mathrm{Ka}$ plan u. Richards (62) geben durchaus positive Erfahrungen mit dieser Methode bei Kindern mit MMC an. Von anderen Arbeitsgruppen wurde diese Methode der intravesikalen Elektrostimulation allerdings mit wenig Erfolg angewandt $(26,34,91,95)$. Boone u. Mitarb. (26) führten eine randomisierte Studie mit einer internen "Sham" behandelten Gruppe $(n=13)$ als Kontrolle zu den über zwei bis drei Wochen transurethral elektrostimulierten 18 Kindern durch. Bei beiden Gruppen fand sich keine subjektive Besserung der Harninkontinenz und der Entwicklung eines Blasenfüllungsempfinden. In der behandelten Gruppe fand sich urodynamisch keine statistisch signifikante $\mathrm{Zu}$ nahme der Blasenkapazität oder der Detrusordruckamplitude.

Die transurethrale Elektrostimulation zum Erlernen eines Miktionsreflexes bei Detrusorareflexie ist nur bei inkompletten Läsionen sinnvoll einzusetzen (76). Sie erfordert eine erhaltene Sensibilität der Blase und damit eine intakte Innervation der Afferenzen, die aber bei neurogener Läsion infolge einer lumbosakralen Meningomyelozele nicht vorausgesetzt werden kann.

Auch die Implantation eines Mentor-Stimulators mit direkter Elektrodenapplikation am Detrusor wurde wegen der unkontrollierten Stromausbreitung, Fibrosierung um die Elektroden und Elektrodendislokation wieder verlassen (84). Die intermittierende Stimulation des Beckenbodens mit Hilfe von anal oder vaginal applizierten Stimulationsprothesen kommt bei den meisten Patienten mit MMC wegen der Denervation des N. pudendus nicht in Frage. Bei Patienten mit intakten Afferenzen des N. pudendus kann durch Stimulation eine Detrusorhyperreflexie inhibiert und der urethrale Verschlußdruck erhöht werden, so daß die Patienten während der Stimulation kontinent sind (51). Ein erheblicher Nachteil dieser Methode besteht darin, daß nicht voraussehbar ist, wie lange der Stimulationseffekt im stimulationsfreien Intervall anhält und die immer wieder notwendige Applikation der Stimulationsprothesen erforderlich ist. 
Tanagho (109) hält in selektionierten Fällen eine funktionelle elektrische Neuromodulation der sakralen Spinalnerven für möglich. Läßt sich bei einer perkutanen Testung der sakralen Spinalnerven allerdings keine Levator-ani-Kontraktion nachweisen, oder ist der Rücken im lumbosakralen Übergang zu vernarbt oder deformiert, kann direkt eine Stimulationstestung des N. pudendus versucht werden, um den Beckenboden zu stimulieren (98). Trotz schlaffer Lähmung der unteren Extremitäten können periphere Nervenfasern intakt sein, da die motorischen Kerne lateral im Rückenmark lokalisiert sind und nicht immer von dem kongenitalen Defekt betroffen sein müssen. Nach Stark (103) sind 90\% der motorischen Vorderhornzellen bei MMC-Patienten intakt. Der Effekt der Neuromodulation der sakralen Spinalnerven oder des N. pudendus wird vor Implantation eines permanenten Stimulationssystems mit einer im Foramen implantierten Elektrode oder der Implantation einer helikalen Elektrode um den N. pudendus und einem telemetrisch programmierbaren Impulsgeber (PISCES Quad Lead, ITREL II, Medtronic Inc. Minneapolis, $M N$ ) durch eine über mehrere Tage durchgeführte Teststimulation mit Hilfe eines perkutan applizierten Schrittmacherdrahtes überprüft. Tanagho (109) berichtete 1992 über 11 von 19 getesteten MMC-Patienten, von denen 7 ein Foramenelektrodenimplantat erhielten. 5 der 7 implantierten Patienten wurden mit der Stimulation vollständig kontinent. Schmidt u. Mitarb. (98) publizierten 1990 zwei Fälle einer erfolgreichen Implantation einer Stimulationselektrode um den N. pudendus (Tab. 8).

Tab. 8 Ergebnisse der funktionellen elektrischen Neuromodulation der sakralen Spinalnerven und des N. pudendus $(98,109)$.

\begin{tabular}{llll}
\hline $\begin{array}{l}\text { Lokalisation der } \\
\text { Stimulations- } \\
\text { elektrode }\end{array}$ & $\begin{array}{l}\text { pos. } \\
\text { perkutane } \\
\text { Testung }\end{array}$ & $\begin{array}{l}\text { erfolgreiche } \\
\text { Implanta- } \\
\text { tion }\end{array}$ & Autoren \\
\hline $\begin{array}{l}\text { sakrale Spinal- } \\
\text { nerven }\end{array}$ & $11 / 19$ & $5 / 7$ & Tanagho, 1992 (109) \\
Nervus pudendus & 2 & Schmidt et al., 1990 (98) \\
\hline
\end{tabular}

Der große Vorteil der beiden Stimulationsmethoden von Schmidt und Tanagho besteht darin, daß die Stimulation ohne Einschränkung über 24 Stunden täglich durchgeführt werden kann und nur bei Miktion unterbrochen wird. Hierzu wird der Impulsgeber vom Patienten mit Hilfe eines Magneten, der über die Implantationstelle geführt wird, ein- und ausgestellt.

\section{Patientenspezifische Komplikations- möglichkeiten bei operativen Eingriffen}

Bei allen operativen Eingriffen bei MMCPatienten ist bei der Planung des operativen Vorgehens zu berücksichtigen, daß bei der überwiegenden Anzahl der Patienten eine schwere Skoliose vorliegt und ein hoher Prozentsatz eine ausgeprägte Latexallergie hat. Insbesondere bei Planung einer harnableitenden Operation mit Urostoma oder einer perkutanen Litholapaxie, muß man daran denken, daß bereits im Kindesalter $50-60 \%$ eine erhebliche Skoliose (zum Teil von mehr als 40 Grad) aufweisen. Das Ausmaß der Skoliose nimmt mit zunehmendem Lebensalter zu. Die Prävalenz korreliert mit der Höhe der
Tab.9 Empfehiungen bei Latex-lgE-Allergie.

intraoperativ keine Latex enthaltenden Materialien benutzen

(latexfreie Op-Handschuhe)

Antihistaminikum 24 Stunden pröoperativ

$\mathrm{H} 1 / \mathrm{H} 2-\mathrm{Blocker}$ präoperativ

Kortikosteroide präoperativ bis zum 4. postoperativen Tag

MMC-Läsion (89). In den letzten Jahren wurde über mehrere schwere intraoperative IgE-vermittelte anaphylaktoide Reaktionen ausgelöst durch eine Latex-Exposition (Allergie Typ I) bei Patienten mit Spina bifida berichtet (83). Es handelte sich dabei überwiegend um Patienten, die bereits über längere Zeit einen intermittierenden Einmalkatheterismus durchführten, mehrere operative Eingriffe hinter sich hatten oder ein Kondomurinal trugen. Bei entsprechender Vorgeschichte sollte ein Radio-allergo-sorbentTest (RAST Test) durchgeführt werden und bei Hinweis auf eine Latex-Allergie eine entsprechende medikamentöse Therapie 24 Stunden vor dem Eingriff eingeleitet werden (Antihistaminikum, H1- und H2-Blocker und Kortikosteroid). Nach Möglichkeit sind alle Latex enthaltenden Materialien während der Operation zu vermeiden (Tab. 9). Latexfreie OP-Handschuhe aus einem thermoplastischen Elastomer (Manex neoderm ${ }^{\circledR}$, Beiersdorf AG, Hamburg) sind seit Juni 1993 erhältlich.

\section{Selektive sakrale Rhizotomie}

Um eine harnableitende Operation bei Kindern mit hohen intravesikalen Drucken zu vermeiden, führten Franco et al. (43) bei 6 Kindern im Alter von 61/2 bis 18 Jahren eine intradurale dorsale Rhizotomie durch. Die Vorderwurzeln wurden in allen Fällen nach intraoperativer Teststimulation identifiziert und geschont. Die Blasenkapazität nahm um durchschnittlich $69 \% \mathrm{zu}$, eine Detrusorhyperreflexie war postoperativ nicht mehr nachzuweisen. Wegen des gleichzeitigen Sensibilitätsausfalles an den unteren Extremitäten steht diese Therapiemöglichkeit bei Patienten mit inkompletten Läsionen und männlichen Patienten mit reflexogenen Erektionen nicht zur Diskussion.

\section{Urolithiasis}

Patienten mit neurogener Blasenfunktionsstörung haben eine erhöhte Prävalenz, Harnsteine zu bilden. Risikofaktoren sind vor allem rezidivierende Harnwegsinfektionen, Immobilisation, Urinstase und ein alkalischer Urin-pH. Die Behandlungsmethode der Wahl ist heute die extrakorporale Stoßwellenlithotrypsie, wobei der Abgang der Desintegrate als Folge der Immobilisation oft verzögert und in vielen Fällen unvollständig ist, so daß einer Rezidivsteinbildung Vorrang geleistet wird (92). Im Einzelfall sollte deshalb insbesondere bei großer Steinmasse eine kombinierte perkutane Nephrolitholapaxie und extrakorporale Stoßwellenlithotrypsie (ESWL)-Behandlung in Erwägung gezogen werden.

Eine lebenslange kontinuierliche urologische Betreuung ist heute eine wesentliche Voraussetzung, um eine sich entwickelnde Nierenfunktionsschädigung rechtzeitig zu diagnostizieren und entsprechende Maß- 
Tab. 10

\begin{tabular}{lll}
\hline $\begin{array}{l}\text { Alter } \\
\text { (Jahre) }\end{array}$ & Untersuchung & $\begin{array}{l}\text { Intervall } \\
\text { (Monate) }\end{array}$ \\
\hline bis 5 & Urinsed./-kultur & 3 \\
& Ultraschall Nieren/RH & 6 \\
& Urodynamik/MZUG & 12 \\
& Nierenszintigraphie & 12 \\
bis 5 & Urinsed./-kultur & 6 \\
& Ultraschall Nieren/RH & 6 \\
& Urodynamik/MZUG & 24 \\
& Nierenszintigraphie & 24 \\
\hline
\end{tabular}

nahmen zu ergreifen. Bei Kindern mit einem "Leak-Point Pressure" $>40 \mathrm{cmH}_{2} \mathrm{O}$, Detrusor-Sphinkter-Dyssynergie oder einem Hostility-Score $>5$ sollte 6 monatlich eine Urinkultur, eine Sonographie beider Nieren, und jährlich eine nuklearmedizinische seitengetrennte Funktionsuntersuchung und eine urodynamische Kontrolle erfolgen. Bei Kindern mit normalem urodynamischen Befund sollte die Urinkultur und die Sonographie beider Nieren jährlich, ein Isotopennephrogramm und eine Kontrollurodynamik alle 2 Jahre durchgeführt werden (Tab. 10). Die urodynamischen Kontrolluntersuchungen sollten in jedem Fall erfolgen, auch wenn neurologisch keine Befundverschlechterung nachzuweisen ist.

In Abhängigkeit von dem bei jedem Kind mit MMC als Basisdiagnostik durchzuführenden Miktionszystourethrogramm, sollte bei nachgewiesenem Reflux einmal jährlich eine röntgenologische Kontrolle veranlaßt werden, bis eventuell eine Spontanmaturation unter der entsprechenden Therapie der neurogenen Blase erfolgt.

\section{Fäkalinkontinenz und Obstipation}

Die Fäkalinkontinenz kann unterteilt werden in eine partiell inkontinente und vollständig inkontinente Form. Um die Stuhlinkontinenz, der verschiedene Ursachen zugrunde liegen können, zu klassifizieren, kann neben der digitalen Untersuchung ein Defäkogramm und eine Rektomanometrie durchgeführt werden. Die Stuhlinkontinenz kann auf einer sensorischen, motorischen oder kombiniert sensorisch-motorischen Innervationsstörung der Anorektalregion, oder einer Störung der Reservoirfunktion der Rektumampulle beruhen. Bei leichteren Formen der Sphinkterinsuffizienz kann eine Elektrostimulation des Levator ani und Sphinkter perinei superficialis und profundus erfolgreich sein. Das Einhalten einer entsprechenden Diät, eine digitale Stimulation, Laxantien, Suppositorien, Einläufe, Analtampons oder die Anwendung spezieller Einlauf-Kontinenz-Katheter (Tab. 11) stehen zur

Tab. 11 Therapiemöglichkeiten bei neurogener intestinaler Dysfunktion.

diätetische Maßnahmen

digitale Stimulation des Darms

Laxantien

Suppositorien

Einläufe \pm Kontinenz-Katheter

PVA-Analstöpsel
Verfügung und sind der individuellen Situation entsprechend anzuwenden $(17,74)$. Analtampons aus PolivinylAlkohol-Schaumstoff stehen in verschiedenen Formen (Konkav-, Zylinder-, Kugeltampon; Med.SSE-System $G m b H$, Fürth) zur Verfügung, und können individuell der Halte- und Abdichtfunktion im Analkanal angepaßt werden. Regelmäßig durchgeführte Einläufe sollen durch Entleerung des Kolon descendens die Fäkalinkontinenz und Obstipation verhindern. 1987 berichteten Shandling u. Gilmour (99) über eine 100\%ige Erfolgsrate bei 112 Kindern mit Spina bifida bei Anwendung eines speziellen Einlauf-Kontinenz-Katheters. Walker u. Webster (115) berichteten über eine $70 \%$ ige Erfolgsrate. Liptak u. Revell (74) modifizierten diesen Ballonkatheter, indem sie die Spritze zum Auffüllen des Ballons im Rektum durch eine leichter $\mathrm{zu}$ bedienende Pumpe ersetzten. Ihre Erfolgsrate betrug $90 \%$, allerdings brachen $50 \%$ der Patienten die Therapie wegen Schmerzen, Unmöglichkeit den Ballon im Rektum zu halten und autonomer Dysregulation ab.

\section{Sexualfunktion und Fertilität}

Der besseren Langzeitbetreuung von Kindern und Jugendlichen mit MMC in den letzten Jahren ist es zu verdanken, daß heute viele dieser Patienten keine eingeschränkte Lebenserwartung mehr haben. Unter diesem Gesichtspunkt stellt sich als neues Problem die Sexualität und Fertilität dieser jungen Erwachsenen dar.

Obwohl die zugrundeliegenden endokrinologischen Mechanismen noch immer unbekannt sind, findet sich bei myelodysplastischen Kindern häufiger als bei gleichaltrigen Mädchen und Knaben eine prämature $\mathrm{Pu}$ bertät $(53,57,85)$. Die sekundären Geschlechtsmerkmale sind bei Mädchen und Jungen nach der Pubertät normal ausgebildet (82). Die Mädchen haben einen normalen Menstruationszyklus, so daß eine Schwangerschaft möglich ist. Allerdings ist nach einer Untersuchung von Carter $u$. Evans (27) das Risiko einer kongenitalen Fehlbildung mit $3 \%$ gegenüber einem normalen Risiko von $0,1 \%$ erhöht. Auch nach Anlage einer kontinenten Harnableitung kann eine Schwangerschaft ausgetragen werden. Es sollten aber in kurzfristigen Abständen regelmäßige Sonographien der Nieren veranlaßt werden, um eine Stauung durch Kompression der Ureteren zu erkennen und die Nieren rechtzeitig zu entlasten.

Die wenigen Angaben zur Sexualität von MMC-Patienten in der Literatur beruhen meistens auf Interviews der Betroffenen. Objektive Untersuchungen und Beobachtungen liegen nicht vor. Shurtleff u. Sousa (102) berichteten, daß nur 5 von 28 befragten Männern mit MMC sexuell aktiv waren und nur einer befriedigende Erektionen angab. Im Gegensatz dazu berichten Diamond u. Mitarb. (35) über Erektionen bei etwa $90 \%$ ihrer MMC-Patienten. $73 \%$ ihrer Patienten hatten nächtliche Emissionen. Etwa $40 \%$ der Männer haben bei Masturbation eine antegrade Ejakulation (102). Allerdings liegen keine objektiven Untersuchungen über Spermiogramme bei Patienten mit MMC vor, so daß keine Aussage über die Fertilität dieser Patienten möglich ist. Die Inzidenz eines Kryptorchismus ist bei Knaben mit MMC mit $25 \%$ signifikant höher, als bei der Normalbevölkerung mit 0,8\% (71). 
In einer Studie von Dorner (36) berichteten 18 von 63 Adoleszenten über eine partnerschaftliche Beziehung, wobei aber nur eine Frau angab, sexuell aktiv zu sein. Nach Shurtleff u. Sousa (102) nimmt der Anteil sexueller Aktivität bei Erwachsenen mit MMC zu. $89 \%$ der Frauen und 29\% der Männer gaben an, regelmäßig sexuelle Kontakte zu haben. Die Erektionsfähigkeit und Ejakulationsfähigkeit sind häufiger erhalten bei Männern mit tiefen, sakralen Läsionen als bei Männern mit hohen Rückenmarksläsionen (30).

Obwohl die ätiologische Zuordnung der erektilen Dysfunktion bei MMC-Patienten schon aufgrund der neurogenen Erkrankung offensichtlich erscheint, sollte auch bei diesen Patienten eine spezifische Basisdiagnostik der Erektionsstörung erfolgen. Sicherlich ist bei einem großen Prozentsatz dieser Patienten mit einer autonomen Innervationsstörung zu rechnen, doch sollten auch reaktive psychogene Faktoren und endokrinologische Ursachen erwogen und untersucht werden. Neben den im Rahmen der Basisuntersuchung erhobenen Laborparametern sollte zusätzlich Prolaktin und Testosteron bestimmt werden. Die Sexualanamnese umfaßt situations- und partnerspezifisches Erektionsverhalten, primäre oder sekundäre Erektionsstörung, Vorhandensein reflexogener Erektionen und Ejakulationen, die Erhebung klassischer Risikofaktoren (Nikotin, Hypertonie, Diabetes, Hypercholesterinämie), die Frage nach Medikamenteneinnahme und nach Voroperationen. Zur Evaluierung einer psychogenen (Mit-)Verursachung sollte ein auf diesem Gebiet erfahrener Psychologe konsultiert werden. Die spezifisch andrologische Diagnostik sollte die Untersuchung der glattmuskulären Funktion der Corpora cavernosa mit Hilfe der standardisierten intrakavernösen Injektion vasoaktiver Substanzen (SKATTestung) einschließen (5). Sie erlaubt Rückschlüsse über die penile Hämodynamik und sollte bei fehlendem Respons wegen einer möglichen psychogenen Inhibition mindestens dreimal wiederholt werden (13). Die intakte Innervation der Corpora cavernosa kann mit Hilfe eines Corpus cavernosum-EMG (CC-EMG) überprüft werden $(48,107)$. Pharmako-Doppler- bzw. Duplexsonographie ermöglichen eine Beurteilung der kavernösen Arterialisation (44). Bei Non-Responder auf die intrakavernöse Injektionstestung sollte zum Ausschluß einer kavernösen Insuffizienz eine Pharmako-Kavernosometrie und -graphie durchgeführt werden (14).

Bei unauffälligen organogenen Befunden und gutem Ansprechen auf die intrakavernöse Injektion vasoaktiver Substanzen und ohne evidente psychogene Ätiologie sollte ein Behandlungsversuch mit Yohimbin (z. B. Yohimbin „Spiegel" ${ }^{\circledR}$, Kali Chemie) begonnen werden. Läßt sich eine autonome Innervationsstörung nachweisen und eine ausreichende Rigidität mit der Schwellkörperinjektion erreichen, sollte der Patient in der Selbstinjektionstechnik unterrichtet werden (106). Voraussetzung für diese Behandlung ist, daß der Patient manuell dazu in der Lage ist und mental so zuverlässig, daß er im Falle einer prolongierten Erektion sich sofort in urologische Behandlung begibt. Da bei Patienten mit neurogener Ätiologie die Gefahr des Auftretens einer prolongierten Erektion prinzipiell höher anzusehen ist, bietet sich als vasoaktive Substanz für die intrakavernöse Injektion der Stickoxid-Donor SIN 1 an, das nach Untersuchungen von Stief et al. (108) im Ge- gensatz zu Papaverin/Phentolamin (Androskat ${ }^{\circledR}, B y k$ Wien) und Prostaglandin (Prostavasin ${ }^{\circledR}$, Schwarz Pharma) nicht zu prolongierten Erektionen führt. Ist der Patient nicht für eine Schwellkörperinjektionstherapie geeignet, bestehen weitere therapeutische Optionen in der Anwendung einer Vakuum-Saug-Hilfe oder der Implantation einer Penisprothese.

\section{Soziale Integration}

Nach einer Untersuchung von Börjeson $u$. Lagergren (21) bei 26 jugendlichen MMC-Patienten im Alter von 15-18 Jahren besteht ein großes Problem in der sozialen Isolation unabhängig vom Grad der Behinderung. Elf Patienten sahen die Inkontinenz als ihr Hauptproblem an. Nur etwa die Hälfte der Patienten war in der Lage sich selbst zu versorgen, wobei dieser Anteil durch praktisches Training und entsprechende Schulung der Eltern sicher zu erhöhen wäre. Alle Jugendlichen hatten ein auffallendes Defizit in der Kenntnis ihres eigenen Körpers und ihrer verschiedenen Behinderungen. Mehrere Untersuchungen haben bestätigt, daß Kinder mit MMC ein signifikant geringeres Selbstbewußtsein und Selbstvertrauen haben $(19,65)$. Eine kontinuierliche Betreuung der Patienten mit MMC vom Schulkind zum Jugendlichen und auch als Erwachsener durch entsprechend geschulte Sozialarbeiter wäre wünschenswert, um diesen Patienten die Integration in unsere Lebensgemeinschaft zu ermöglichen und zu erleichtern. In Schweden stehen für Personen mit motorischer Behinderung und normaler Intelligenz sogenannte "Handicapped Adult Teams (HAT)" zur Verfügung, die die Arbeit der "Handicapped Children Teams" fortsetzen, und sich um die weitere Ausbildung, soziale und medizinische Absicherung kümmern (21).

\section{Kommentar}

„Nur 6,5\% aller Kinder werden spontan kontinent (30) und $40 \%$ haben einen pathologischen urethralen Auslaßwiderstand von über $40 \mathrm{cmH}_{2} \mathrm{O}$ und zählen damit zur renal gefährdeten Gruppe." Zweifellos führt der heute bereits prophylaktisch angewandte CIC zu einer signifikanten Verminderung von Harnwegsinfekten und damit zur Erhaltung der Nierenfunktion $(113,63)$.

Solange die Kinder unter elterlicher Obsorge sind und die lokale Anwendung von Anticholinergika ebenso gewährleistet ist wie die antibiotische Prophylaxe, ist insbesondere bei Mädchen die Prognose günstig. Mit fortschreitendem Lebensalter und abnehmender Compliance überwiegt das Problem der Inkontinenz, wie die Untersuchungen von Börgeson et al. (2) am Ende der Arbeit zeigen. Diese entsprechen im vollen Umfang unseren eigenen Erfahrungen, wobei die Patienten mit thorakaler Läsion und überwiegend rollstuhlpflichtig im Vordergrund stehen. Spätestens mit Abschluß der Berufsausbildung und der ersten Partnerschaft wird von diesen Patienten die Frage nach einer Alternative zum technisch schwieriger werdenden CIC immer häufiger gestellt.

Der Autor verweist auf die Untersuchungen von Benson (15), der die Komplikationen des IleumConduits $(\mathrm{n}=51)$ mit denen des Ileozökalpouches $(\mathrm{n}=22)$ verglich und keinen signifikanten Unterschied fand. Bei 
dem vorgestellten Krankengut handelt es sich um $43 \mathrm{~Pa}$ tienten mit Blasenkarzinomen, bei denen gleichzeitig eine Zysto-Prostatektomie durchgeführt wurde, bei den restlichen vorwiegend um bestrahlte Patienten mit Beckenmalignomen. Dementsprechend überwiegen in den aufgelisteten Früh- und Spätkomplikationen für das onkologische Krankengut Relevante von 50-60jährigen, wobei kardiovaskuläre Bestrahlungs- und infektionsbedingte Komplikationen überwiegen. Im Gegensatz dazu dominieren bei Kindern die oberen Harnwege, wobei der $\mathrm{Zu}$ stand der Ureteren eine entscheidende Rolle spielt. Unabhängig davon betonen die Autoren die ausgezeichnete Kontinenzrate und die niedrige Komplikationsrate von seiten der oberen Harnwege und dies trifft auch für MMCPatienten zu, für die ein nasses Stoma von vorneherein inakzeptabel ist.

Bezüglich der hyperchlorämischen metabolischen Azidose mit Osteomalazie $(69,79)$ ist der prophylaktische Ausgleich des negativen Base excess von Werten $>-2,5$ entscheidend. So findet sich in der von Koch zitierten Arbeit folgender Hinweis:

„Wir haben anhand von Rattenversuchen gezeigt, daß Bikarbonatgabe die Demineralisation verhindert, was möglicherweise bei Menschen ebenfalls der Fall sein könnte."Während in Europa diese prophylaktische Therapie standardisiert ist und regelmäßige Astrupkontrollen eine Conditio sine qua non sind, wird in USA erst zu einem späteren Zeitpunkt die „hyperchlorämische Azidose“ behandelt. Dieser Unterschied ist essentiell und erklärt die von Koch gefundenen Wachstumsunterschiede von Kindern mit CIC im Gegensatz zum Ileum-Conduit.

In der Arbeit von Cass 1984 werden die bekannten Komplikationen des Ileum-Conduits beschrieben und mit Recht schließt der Autor daraus, daß auf diese Form der Harnableitung verzichtet werden sollte. Im letzten Absatz der Arbeit von Cass werden die bis zu $60 \%$ igen Nierenfunktionsverschlechterungen des Ileum-Conduits mit den $60 \%$ igen des Kolon-Conduits aus der Arbeit von Elder 1979 verglichen.

Hierbei übersehen sowohl Cass als auch der Autor in seiner Übersichtsarbeit, daß es sich bei dem von Elder nachuntersuchten Krankengut von Moog in Cardiff keineswegs um eine antirefluxive Ureterimplantation handelte. Vielmehr erwiesen sich die anisoperistaltisch ausgeschalteten und mit einem Stomanippel versehenen Conduits als Hochdruckreservoire und die Ureternippel als refluxiv. Dies im Gegensatz zu den isoperistaltischen und submukös implantierten Ureteren mit flachem nicht obstruktivem Stoma, erstmals von Übelhor 1951 publiziert und später von anderen (H. Hendren, Altwein) übernommen.

Zusammenfassend ist der Autor zu seiner zweiteiligen Übersichtsarbeit, die den heutigen diagnostischen und therapeutischen Kenntnisstand widergibt, zu beglückwünschen. Vor dem Hintergrund einer deutlich höheren Überlebenschance aufgrund verminderter renaler Komplikation wächst die Zahl von Patienten, bei denen mit fortschreitendem Lebensalter das „Kontinenzproblem" vordergründig wird. Hierbei wird die erreichte „so- ziale Kontinenz“ vom Patienten oftmals als Inkontinenz empfunden.

In den kommenden Jahren wird man sich dieser Herausforderung stellen müssen, wie die stetig zunehmende Zahl von Patienten, die eine Konversion in eine kontinente Harnableitung wünschen, zeigt. Voraussetzung ist eine sorgfältige Analyse aller bekannten Komplikationen der verschiedenen Harnableitungsverfahren und deren Ursache. Oftmals sind es scheinbar unwesentliche Details, die über Erfolg oder Mißerfolg entscheiden, darauf hinzuweisen ist der Zweck dieses Kommentars.

\section{R. Hohenfellner, Mainz}

\section{Kommentar}

Bezüglich einer Basisdiagnostik zum Problem der erektilen Dysfunktion bei Patienten mit Meningomyelozele sind hormonelle Untersuchungen nur dann sinnvoll, wenn inspektorisch oder von der Untersuchung her Hinweise für eine Störung in der Hormonachse bestehen (z.B. Kryptorchismus). Die Einbeziehung eines Psychologen ist primär sicherlich nicht nötig, sondern nur dann gerechtfertigt, wenn objektive Tests eine organische Läsion ausschließen. Auch das Corpus-cavernosum-EMG halte ich für eine in der Routineabklärung der ED noch nicht etablierte Methode, die gerade bei diesem Patientengut sicherlich noch experimentellen Charakter besitzt. Therapeutische Versuche mit Yohimbin besitzen wohl eher Plazebo-Charakter und könnten allenfalls der psychiatrischen Therapie vorgeschaltet werden. Die übrigen diagnostischen Möglichkeiten sollten nur so weit vorangetrieben werden, bis sie zur Diagnosestellung bezüglich der Ursachen der ED nötig sind. Individuelle Wünsche und Gegebenheiten des Patienten sind dabei zu berücksichtigen.

S. C. Müller, Mainz

\section{Literaturverzeichnis}

${ }^{1}$ Adams, M. C., M. E. Mitchell, R. C. Rink: Gastrocystoplasty: an alternative solution to the problem of urological reconstruction in the severely compromised patient. J. Urol. 140 (1988) 1152-1156

2 Amark, $P$., A. Nergardh: Influence of adrenergic agonists and antagonists on urethral pressure, bladder pressure and detrusor hyperactivity in children with myelodysplasia. Acta. Paediatr. Scand. 80 (1991) 824-832

3 Andersen. J. T.: Pharmacological treatment of incontinence and voiding disorders. Current Opinion in Urology 2 (1992) $273-276$

${ }^{4}$ Atala, A., S. B. Bauer, W. H. Hendren, A. B. Retik: The effect of gastric augmentation on bladder function. J. Urol. 149 (1993) 1099-1102

5 Bähren, W. C. G. Stief, W. Scherb, H. Gall, A. Gallwitz, J. Altwein: Rationelle Diagnostik der erektilen Dysfunktion unter Anwendung eines pharmakologischen Tests. Akt. Urol. 17 (1986) 177-180

6 Barrett, D. M., B. G. Parulkar: The artificial sphincter (AS 800). Experience in children and young adults. Urol. Clin. North Am. 16 (1989) 119-132

7 Baskin, L. S., B. A. Kogan, F. Benard: Treatment of infants with neurogenic bladder dysfunction using anticholinergic drugs and intermittent catheterisation. Brit. J. Urol. 66 (1990) $532-534$ 
${ }^{8}$ Bauer, S. B., M. Hallett, S. Khoshbin, R. L. Lebowitz, K. R. Winston, S. Gibson, A. H. Colodny, A. B. Retik: Predictive value of urodynamic evaluation in newborns with myelodysplasia. J.A.M.A. 252 (1984) 650-654

${ }^{9}$ Bauer, S. B., E. F. Redd, A. H. Colodny, A. B. Retik: Detrusor instability, a delayed complication in association with the artifical sphincter. J. Urol. 135 (1986) 1212-1215

${ }^{10}$ Bauer, $S$. B.: Urologic management of the myelodysplastic child. Prob. Urol. 3 (1989) 86-101

11 Bauer, S. B., W. H. Hendren, H. Kozakewich, S. Maloney, A. H. Colodny, J. Mandell, A. B. Retik: Perforation of the augmented bladder. J. Urol. 148 (1992) 699-703

12 Bellinger, M. F.: Ureterocystoplasty: a unique method for vesical augmentation in children. J. Urol. 149 (1993) 811-813

${ }^{13}$ Benard, F, C. G. Stief, R. Bosch, S. Aboseif, T. F. Lue, E. A. Tanagho: The effect of epinephrine on erection. J. Urol. 141 (1989) 439 a

14 Bennett, A. H., F. A. Garofalo: Enhanced cavernometry: procedure for the diagnosis of venogenic impotence. Br. J. Urol. 64 (1989) 420-422

15 Benson, $M$. . C., K. M. Slawin, $M . H$. Wechsler, C. A. Olsson: Analysis of continent versus standard urinary diversion. Br. J. Urol. 69 (1992) 156-162

16 Bitscher, M., H. Nerstrom, J. Nordling, T. Hald: Upper urinary tract deterioration after implantation of artificial urinary sphincter. Scand. J. Urol. Nephrol. 24/1 (1990) 31-34

17 Blair, G. K., K. Djonlic, G. C. Fraser, W. D. Arnold, J. J. Murphy, $B$. Irwin: The bowel management tube: an effective means for controlling fecal incontinence. J. Pediatr. Surg. 27 (1992) 1269-1272

18 Bloom, D. A., J. M. Knechtel, E. J. McGuire: Urethral dilatation improves bladder compliance in children with myelomeningocele and high leak point pressure. J. Urol. 144 (1990) 430-433

${ }^{19}$ Blum, R. W. The adolescent with spina bifida. Clinical Pediatrics 22 (1983) $331-335$

${ }^{20}$ Blyth, B., D. H. Ewalt, J. W. Duckett, H. M. Snyder, III.: Lithogenic properties of enterocystoplasty. J. Urol. 148 (1992) $575-577$

21 Börjeson, M.-C., J. Lagergren: Live conditions of adolescents with myelomeningocele. Dev. Med. Child. Neurol. 32 (1990) 698-706

22 Borzi, P. A., J. Bruce, D. C. S. Gough: Continent cutaneous diversions in children: Experience with the Mitrofanoff Procedure. Br. J. Urol. 70 (1992) 669-673

23 Bramble, $F$. J.: The treatment of adult enuresis and urge-incontinence by enterocystoplasty. Brit. J. Urol. 54 (1982) 693-696

${ }^{24}$ Brendler, C. B., L. C. Radebaugh, J. L. Mohler: Topical oxybutynin chloride for relaxation of dysfunctional bladders. J. Urol. 141 (1989) 1350-1352

25 Bricker, E. M.: Bladder substitution after pelvic evisceration. Surg. Clin. North. Amer. 30 (1950) 1511-1514

26 Boone, T. B., C. G. Roehrborn, G. Hurt, G.: Transurethral intravesical electrotherapy for neurogenic bladder dysfunction in children with myelodysplasia: a prospective randomized clinical trial. J. Urol. 148 (1992) 550-554

27 Carter, $C$. $O$., K. Evans: Children of adult survivors with spina bifida cystica. lancet 2 (1973) 924-926

28 Cartwright, P. C., B. W. Snow: Bladder autoaugmentation: early clinical experience. J. Urol. 142 (1989) 505-508

${ }^{29}$ Cass, A. S., M. Luxenberg, P. Gleich, C. F. Johnson: A 22-year followup of ileal conduits in children with a neurogenic bladder. J. Urol. 132 (1984) 529-531

${ }^{30}$ Cass, A. S., B. A. Bloom, M. Luxenberg: Sexual function in adults with myelomeningocele. J. Urol. 136 (1986) 425-426

31 Cher, M. L., T. D. Allen: Continence in the myelodysplastic patient following enterocystoplasty. J. Urol. 149 (1993) 1103-1106

${ }^{32}$ Crane, J. M., H. S. Scherz, G. F. Bilman, G. W. Kaplan: Ischemic necrosis: a hypothesis to explain the pathogenesis of sponta- neously ruptured enterocystoplasty. J. Urol. 146 (1991) $141-144$

${ }^{33}$ Dalkin, B., I. Franco, E. F. Reda, D. McLone, L. Godine, W. E. Kaplan: Contrast-induced central nervous system toxicity after radiographic evaluation of the lower urinary tract in myelodysplastic patients with ventriculoperitoneal shunts. J. Urol. 148 (1992) 120-121

${ }^{34}$ Decter, R. M. P. Synder, T. K. Rosvanis: Transurethral electrical bladder stimulation: initial results. J. Urol. 148 (1992) 651-653

35 Diamond, D. A., A. M. Rickwood, D. G. Thomas: Penile erections in meningomyelocele patients. Brit. J. Urol. 58 (1986) 434-435

36 Dorner, $S$.: Sexual interest and activity in adolescents with spina bifida. J. Child. Psychol. Psychiatry. 18 (1977) 229-237

37 Duckett, $J$. W. Jr. Cutaneous vesicostomy in childhood. The Blocksom technique. Urol. Clin. North Am. 1 (1974) 485-495

${ }^{38}$ Dykes, E. H., P. G. Ransley: Gastrocystoplasty in children. Br. J. Urol. 69 (1992) 314-317

${ }^{39}$ Elder, J. S., H. M. Synder, W. C. Hulbert, J. W. Duckett: Perforation of the augmented bladder in patients undergoing clean intermittent catheterization. J. Urol. 140 (1988) 1159-1162

40 Elder, J. S.: Continent appendicocolostomy: a variation of the Mitrofanoff principle in pediatric urinary tract reconstruction. J. Urol. 148 (1992) 117-119

41 Fenn, N., I. G. Conn, K. A. German, T. P. Stephenson: Complications of clam enterocystoplasty with particular reference to urinary tract infection. Br. J. Urol. 69 (1992) 366-368

42 Filmer, R. B., J. R. Spencer: Malignancies in bladder augmentations and intestinal conduits. J. Urol. 143 (1990) 671-678

43 Franco, I., B. Storrs, C. F. Firlit, K. Zebold, I. Richards, W. E. Kaplan: Selective sacral rhizotomy in children with high pressure neurogenic bladders: preliminary results. J. Urol. 148 (1992) 648-650

44 Gall, H., W. Bähren, W. Scherb, C. Stief, W. Thon: Diagnostic accuracy of Doppler ultrasound technique of the penile arteries in correlation to selective arteriography. Cardiovasc. Intervent. Radiol. 11 (1988) 225-231

45 Galloway, N. T. M., J. A. Mekras, M. Helms, G. B. Weber: An objective score to predict upper tract deterioration in myelodysplasia. J. Urol. 145 (1991) 535-537

46 Gearhart, J. P., P. C. Albertsen, E. F. Marshall, R. B. Jeffs: Pediatric applications of augmentation cystoplasty: The John Hopkins Experience. J. Urol. 136 (1986) 430-432

47 Geraniotis, E., S. A. Koff, B. Enrile: The prophylactic use of clean intermittent catheterization in the treatment of infants and young children with myelomeningocele and neurogenic bladder dysfunction. J. Urol. 139 (1988) 85-86

48 Gerstenberg, T. C., J. Nordling, H. Hald, G. Wagner: Standardized evaluation of erectile dysfunction in 95 consecutive patients. J. Urol. 141 (1989) 857-862

49 Ghoniem, G. M., M. B. Roach, V. H. Lewis, E. P. Harmon: The value of leak point pressure and bladder compliance in the urodynamic evaluation of meningomyelocele patients. J. Urol. 144 (1990) 1440-1442

50 Gilchrist, $R$. K., et al.: Construction of a substitute bladder and urethra. Surg. Gynecol. Obstet. 90 (1950) 752-756

51 Godec, C. J., A. S. Cass: Electrical stimulation for incontinence in myelomeningocele. J. Urol. 120 (1978) 729-731

52 Gold, B. D., P. S. Bhoopalam, R. M. Reifen, E. Harvey, M. A. Marcon: Gastrointestinal complications of gastrocystoplasty. Arch. Dis. Child. 67 (1992) 1272-1276

53 Greene, S. A., M. Frank, M. Zachmann, A. Prader: Growth and sexual development in children with myelomeningocele. Eur. J. Pediatr. 144 (1985) 146-148

${ }^{54}$ Greenfield, S. P., M. Fera: The use of intravesical oxybutyninchlorid in children with neurogenic bladder. J. Urol. 146 (1991) $532-534$

55 Hannigan, $K$., J. Elder: Teaching catheterization to children. Urol. Clin. North. Am. 15 (1988) 653-660

56 Harzmann, R.: Karzinomrisiko nach Harnableitung. Akt. Urol. 24 (1993) 63-64 
${ }^{57}$ Hayden, P. W., S. L. Davenport, M. M. Campbell: Adolescents with myelodysplasia: impact on physical disability on emotional maturation. Pediatrics 64 (1979) 53-59

58 Johnston, J. H., B. L. Kathel: The obstructed neurogenic bladder in the newborn. Brit. J. Urol. 43 (1971) 206-210

59 Joseph, D. B., S. B. Bauer, A. H. Colodny, J. Mandell, A. B. Re $t i k$ : Clean intermittent catheterization of infants with neurogenic bladder. Pediatrics 84 (1989) 78-82

60 Kaplan, W. E.: Management of myelomeningocele. Urol. Clin. N. Am. 12 (1985) 93-101

${ }^{61}$ Kaplan, W. E., C. F. Firlit: Management of reflux in the myelodysplastic child. J. Urol. 129 (1983) 1195-1197

62 Kaplan, W. E., I. Richards: Intravesical transurethral electrotherapy for the neurogenic bladder. J. Urol. 136 (1986) $243-246$

63 Kasabian, N. G., S. B. Bauer, F. M. Dyro, A. H. Colodny, J. Mandell, A. B. Retik: The prophylactic value of clean intermittent catheterization and anticholinergic medication in newborns and infants with myelodysplasia at risk of developing urinary tract deterioration. Amer. J. Dis. Child. 146 (1992) 840-843

64 Katona, F. $M$. Berenyi, $M$.: Intravesical transurethral electrotherapy in meningomyelocele patients. Acta Paediatr. Acad. Sci. Hung. 16 (1975) 363-374

${ }^{65} \mathrm{Kazak}, \mathrm{A}$. E., M. W. Clark: Stress in families of children with myelomeningocele. Dev. Med. Child. Neurol. 28 (1986) $220-228$

${ }^{66}$ Keating, M. A., R. C. Rink, M. C. Adams: Appendicovesicostomy: a useful adjunct to continent reconstruction of the bladder. J. Urol. 149 (1993) 1091-1094

67 Khoury, J. M., G. D. Webster: Evaluation of augmentation cystoplasty for severe neuropathic bladder using the hostility score. Developmental Medicine and Child Neurology 34 (1992) $441-447$

${ }^{68}$ Kinahan, T. J., A. E. Khoury, G. A. McLorie, B. M. Churchill: Omeprazole in post-gastrocystoplasty metabolic alkalosis and aciduria. J. Urol. 147 (1992) 435-437

${ }^{69}$ Koch, M. O., W. S. McDougal, M. C. Hall, D. E. Hill, H. V. Braren, $M$. N. Donofrio: Long-term metabolic effects of urinary diversion: a comparison of myelomeningocele patients managed by clean intermittent catheterization and urinary diversion. J. Urol. 147 (1992) 1343-1347

${ }^{70}$ Kock, N. G., L. O. Nilsson, L. J. Norlen, B. M. Philipson: Urinary diversion by a continent ileal reservoir: Clinical results in 12 patients. J. Urol. 128 (1982) 469-475

71 Kropp, K. A., K. K. S. Voeller: Cryptochidism in myelomeningocele. J. Pediatr. $99^{\circ}(1981)$ 110-113

72 Lapides, J., A. C. Diokno, S. J. Silber, B. S. Lowe: Clean, intermittent self-catheterization in the treatment of urinary tract disease. J. Urol. 107 (1972) 458-461

${ }^{73}$ Lindehall, B., I. Claesson, K. Hjalmas, U. Jodal: Effect of clean intermittent catheterisation on radiological appearance of the upper urinary tract in children with meningomyelocele. Brit. J. Urol. 67 (1991) 415-419

74 Liptak, G. S., G. M. Revell: Management of bowel dysfunction in children with spinal cord disease or injury by means of the enema continence catheter. J. Pediatr. 120 (1992) 190-194

75 Lockhart, J. L., D. Bejany, V. A. Politano: Augmentation cystoplasty in the management of neurogenic bladder diesease and incontinence. J. Urol. 135 (1986) 969-971

${ }^{76}$ Madersbacher, H., W. Pauer, E. Reiner: Rehabilitation of micturition by transurethral electrostimulation of the bladder in patients with incomplete spinal cord lesions. Paraplegia 20 (1982) 191-195

77 Mandell, J., S. B. Bauer, A. H. Colodny, A. B. Retik: Cutaneous vesicostomy in infancy. J. Urol. 126 (1981) 92-93

${ }^{78}$ Massad, C. A., B. A. Kogan, F. E. Trigo-Rocha: The pharmacokinetics of intravesical and oral oxybutynin chloride. J. Urol. 148 (1992) 595-597

${ }^{79} \mathrm{McDougal}$, W. S.: Metabolic complications of urinary intestinal diversion. J. Urol. 147 (1992) 1199-1208

${ }^{80}$ McGuire, E. J., J. R. Woodside, T. A. Carclen, R. M. Weiss: Pro- gnostic value of urodynamic testing in myelodysplastic patients. J. Urol. 126 (1981) 205-209

81 McGuire, E. J.: Immediate Management of the inability to void, in: Practical urology in spinal cord injury, eds. Parsons, $K . F, J$. $M$. Fitzpatrick, Springer Verlag (1991) 5-10

82 McGuire, E. J., J. Denil: Adult myelodysplasia. AUA Update Series 10/38 (1991) 298-303

83 Merguerian, P. A., R. B. Klein, M. A. Graven, A. A. Rozycki: Intraoperative anaphylactic reaction due to Latex hypersensitivity. Urology 38 (1991) $301-304$

84 Merrill, D. C.: Clinical experience with the mentor bladder stimulator. II. Meningomyelocele patients. J. Urol. 112 (1974) $823-825$

85 Meyer, S., H. Landau: Precocious puberty in myelomeningocele patients. J. Pediatr. Orthop. 4 (1984) 28-31

86 Mitchell, M. E., J. A. Piser: Intestinocystoplasty and total bladder replacement in children and young adults: followup in 129 cases. J. Urol. 138 (1987) 579-584

87 Mitrofanoff, P.: Cystostomie continente trans-appendiculaire dans le traitement des vessies neurologiques. Chir. Ped. 21 (1980) 297-305

${ }^{88} \mathrm{Mogg}, R$. A.: The treatment of neurogenic urinary incontinence using the colonic conduit. Br. J. Urol. 37 (1965) 681-684

${ }^{89}$ Müller, E. B. A. Nordwall: Prevalence of scoliosis in children with myelomeningocele in western Sweden. Spine 17/9 (1992) 1097-1102

${ }^{90}$ Mundy. A. R., D. E. Nurse: Calcium balance, growth, skeletal mineralization in patients with cystoplasties. Br. J. Urol. 69 (1992) 257-259

91 Nicholas, J. L., H. B. Eckstein: Endovesical electrotherapy in treatment of urinary incontinence in spina bifida patients. Lancet 2 (1975) 1276-1277

92 Niedrach, W. L., R. S. Davis, F. W. Tonetti, A. T. K. Cockett: Extracorporal shock-wave lithotripsy in patients with spinal cord dysfunction. Urology 38 (1991) 152-156

93 Perez, L. M., J. Khoury, G. D. Webster: The value of urodynamic studies in infants less than 1 year old with congenital spinal dysraphism. J. Urol. 148 (1992) 584-587

94 Perkash, I., J. Giroux: Clean intermittent catheterization in spinal cord injury patients: a followup study. J. Urol. 149 (1993) $1068-1071$

95 Petersen, T.: Management of urinary incontinence in children with meningomyeleocele. Acta Neurol. Scand. 75/1 (1987) 5255

96 Reinberg, Y., J. C. Manivel, C. Froemming, R. Gonzalez: Perforation of the gastric segment of an augmented bladder secondary to peptic ulcer disease. J. Urol. 148 (1992) 369-371

97 Rowland, R. G., M. E. Mitchell, R. Bihrle, R. J. Kahnoski, J. E. Piser: Indiana continent urinary reservoir. J. Urol. 137 (1987) $1136-1139$

98 Schmidt, R. A., B. A. Kogan, E. A. Tanagho: Neuroprostheses in the management of incontinence in myelomeningocele patients. J. Urol. 143 (1990) 779-782

99 Shandling, B., R. F. Gilmour: The anal sphincter force in health and disease. J. Pediatr. Surg. 22 (1987) 754-757

100 Sheiner, J. L., G. W. Kaplan: Spontaneous bladder rupture following enterocystoplasty. J. Urol. 140 (1988) 1157-1158

101 Shochat, S. J., A. D. Perlmutter: Myelodysplasia with severe neonatal hydronephrosis: the value of urethral dilatation. J. Urol. $107 / 1972)$ 146-148

102 Shurtleff. D. B., J. C. Sousa: The adolescent male with myelodysplasia, development, achievement, sex, and deterioration. Del. Med. J. 49 (1977) 631-638

103 Stark, G. D.: The nature and cause of paraplegia in myelomeningocele. Paraplegia 9 (1972) 219-223

104 Steiner, M. S., R. A. Morton. F. F. Marshall: Vitamin B12 deficiency in patients with ileocolic neobladders. J. Urol. 149 (1993) 255-257

105 Stephenson, T. P.: Artificial urinary sphincters. Current Opinion in Urology, 3/3 (1993) 182-185

106 Stief, C. G., W. Bähren, H. Gall, W. Scherb, A. Gallwitz, J. E. Alt- 
wein: Schwellkörper-Autoinjektionstherapie (SKAT): erste Erfahrungen bei erektiler Dysfunktion. Urologe A, 25 (1986) $63-66$

107 Stief, C. G., M. Djamilian, P. Anton, E. P. Allhoff, U. Jonas: Single potential analysis of cavernous electric activity in impotent patients: a possible diagnostic method for autonomic cavernous dysfunction and cavernous smooth muscle degeneration. J. Urol. 146 (1991) 771-776

108 Stief, C. G., F. Holmquist, M. Djamilian, H. Krah, K. E. Andersson, $U$. Jonas: Preliminary results with the nitric oxide donor linsidomine cholalhydrate in the treatment of human erectile dysfunction. J. Urol. 148 (1992) 1437-1440

109 Tanagho, E. A.: Neuromodulation in management of voiding dysfunction in children. J. Urol. 148 (1992) 655-657

110 Thirumavalan, V. S., P. G. Ransley: Epididymitis in children and adolescents on clean intermittent catheterization. Eur. Urol. 22 (1992) 53-56

111 Thüroff, J. W., P. Alken, U. Engelmann, H. Riedmiller, G. H. Jacobi, $R$. Hohenfellner: Der Mainz-Pouch zur Blasenerweiterungsplastik und kontinenten Harnableitung. Akt. Urol. 16 (1985) $1-8$

112 Thüroff, J. W. B. Bunke, A. Ebner, P. Faber, P. De Geeter, J. Hannapel, H. Heidler, H. Madersbacher, W. Schäfer, et al.: Randomized, double blind, multicenter trial on treatment of frequency, urgency and incontinence related to detrusor hyperactivity: oxybutynin versus propantheline versus placebo. J. Urol. 145 (1991) 813-817

113 Van Gool, J. D., T. P. V. M. de Jong, T. M. Boemers: Einfluß des intermittierenden Katheterismus auf Harnwegsinfekte und Inkontinenz bei Kindern mit Spina bifida. Monatsschr. Kinderheilkd. 139 (1991) 592-596

114 Wagstaff, K. E., C. R. J. Woodhouse, P. G. Duffy, P. G. Ransley: Delayed linear growth in children with enterocystoplasties. Br. J. Urol. 69 (1992) 314-317

115 Walker, J., P. Webster: Successful management of faecal incontinence using the enema continence catheter. Z. Kinderchir. 44, suppl. 1 (1989) 44-45

116 Wang, S. C., E. J. McGuire, D. A. Bloom: Urethral dilatation in the management of urological complications of myelodysplasia. J. Urol. 142 (1989) 1054-1055

117 Wolf, J. S., C. W. Turzan:Augmentation ureterocystoplasty. J. Urol. 149 (1993) 1095-1098
PD Dr. med. Walter F. Thon

Urologische Klinik

Medizinische Hochschule Hannover

Konstanty-Gutschow-Str. 8

30625 Hannover 